\title{
Castration-Resistant Prostate Carcinoma
}

National Cancer Institute

\section{Source}

National Cancer Institute. Castration-Resistant Prostate Carcinoma. NCI Thesaurus.

Code C130234.

Prostate carcinoma that grows and continues to spread despite the surgical removal of the testes or medical intervention to block androgen production. 